\title{
Decoloring Methyl Orange under Sunlight by a Photocatalytic Membrane Reactor Based on ZnO Nanoparticles and Polypropylene Macroporous Membrane
}

\author{
Bing $\mathrm{Hu},{ }^{1,2}$ Jin $\mathrm{Zhou},{ }^{1,2}$ and Xiu-Min $\mathrm{Wu}^{2}$ \\ ${ }^{1}$ Department of Material and Chemistry Engineering, Chizhou University, Chizhou, Anhui 247000, China \\ ${ }^{2}$ Laboratory of Functional Molecular Solids, Ministry of Education, Anhui Key Laboratory of Molecular-Based Materials, \\ College of Chemistry and Materials Science, Anhui Normal University, 1 East Beijing Road, Wuhu, Anhui 241000, China
}

Correspondence should be addressed to Jin Zhou; zhoujin_ah@163.com

Received 2 February 2013; Accepted 10 March 2013

Academic Editor: Hai-Yin Yu

Copyright (C) 2013 Bing Hu et al. This is an open access article distributed under the Creative Commons Attribution License, which permits unrestricted use, distribution, and reproduction in any medium, provided the original work is properly cited.

\begin{abstract}
Decoloring methyl orange (MeOr) under sunlight was conducted in a photocatalytic membrane reactor (PMR). Zinc oxide nanoparticles ( $\mathrm{ZnO}$ NPs) were suspended in the solution or immobilized on the membrane. The membrane was modified by grafting 2-hydroxyethyl methacrylate (HEMA) to enhance the adsorption of ZnO NPs on the hydrophobic membrane surface and improve the membrane permeability. The results show that the water fluxes through the modified membranes are higher than that through the unmodified membrane. After introducing $\mathrm{ZnO} N$ ss to the membrane, the water fluxes still rise with the immobilization degree of ZnO NPs. For the PMR with ZnO NPs in suspension, the photocatalytic decoloration percent (PDP) was over 98.2\% after 40 min under sunlight. For the PMR with ZnO NPs immobilized on the membrane, the max of PDP was $74.3 \%$ after $6 \mathrm{~h}$ under sunlight, and maintained at $72 \%$ after repeated uses for five times. These results demonstrate that photocatalytic membrane reactor (PMR) based on $\mathrm{ZnO}$ NPs and polypropylene macroporous membrane(PPMM) could be applied in decoloring dyes.
\end{abstract}

\section{Introduction}

Dyes, widely used in industries, like textiles, paper, rubber, and plastics, have led to severe environmental contamination due to the toxic and colored wastewater poured into water bodies, which seriously worsens the quality of water, inhibiting sunlight penetration and reducing photosynthetic reaction.

Conventional treatment methods for dye removal, such as ozonation, bleaching, hydrogen peroxide/UV, and electrochemical techniques, were found to be nondestructive and inefficient, because most dyes have complex aromatic molecular structures that resist degradation [1]. They are stable to light, oxidizing agents, and aerobic digestion. The motivation for more efficient treatment processes has inspired environmental scientists and engineers to explore the technique of combing pressure-driven membrane filtration and heterogeneous photocatalysis [2-6].
Recently, heterogeneous photocatalysis employing semiconductor photocatalysts $\left(\mathrm{TiO}_{2}, \mathrm{ZnO}\right.$, and adulterate or complex semiconductor) has demonstrated their efficiencies in degrading a wide range of toxic organic compounds into relatively innocuous end products, such as carbon dioxide and water [5]. Photocatalytic oxidation of various harmful organic dyes and inorganic pollutants in industrial wastewater has been carried out by nanosemiconductors under UV light due to their high photosensitivity and nontoxicity $[7,8]$.

$\mathrm{ZnONPs}$ have great photocatalytic activity under sunlight, which can effectively absorb photons in the UV region from the sunlight [9] and have shown higher photocatalytic efficiencies for the degradation of several organic pollutants $[10,11]$. It is known that many adulterate or complex semiconductors have shown great photocatalytic activity under solar light $[12,13]$. Compared with these semiconductors, the advantages of ZnO NPs are simple synthesis process (only one step by chemical depositing method) and low cost. 


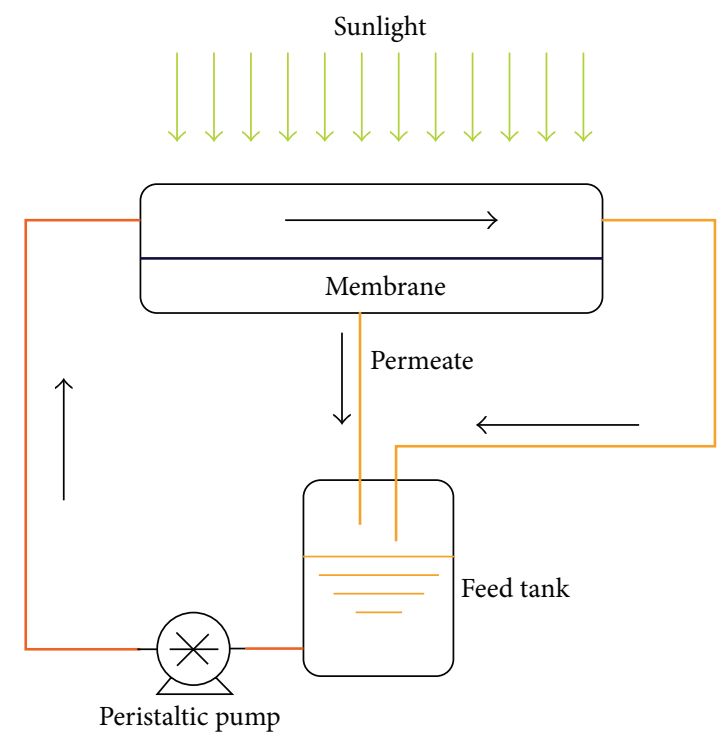

FIGURE 1: A schematic diagram of the photocatalytic membrane reactor (active membrane area is $2700 \mathrm{~mm}^{2}$ ).

On the other hand, membrane process is widely applied in many industries. The process has several main advantages, such as low energy consumption, low chemicals consumption, low maintenance costs, and easy scale up [14].

Photocatalytic membrane reactors (PMRs) are of hybrid processes in which photocatalysis is coupled with a membrane process, and the permeation of solutes through the membrane and photocatalytic reaction occurs simultaneously. As a low-cost, environmentally friendly, and sustainable treatment technology, the PMRs for wastewater treatment using semiconductors have shown a great potential $[15,16]$. Although UV light has been commonly employed in the photocatalytic studies, worldwide efforts are underway to make use of sunlight for environmental protection and water purification [17], because sunlight consists of about 5-7\% UV light, $46 \%$ visible light, and $47 \%$ other irradiation [18], and additionally it is inexhaustible (Figure 1).

In the present work, to improve the membrane permeability and to enhance the adsorption of the semiconductors, hydrophilic surface modification of polypropylene macroporous membrane was carried out. Grafting 2-hydroxyethyl methacrylate (HEMA) onto the membrane surface was performed according to previous work $[19,20]$. $\mathrm{ZnO}$ NPs were one pot fabricated by direct precipitation; their photocatalytic activity for environmental application was carefully investigated by using decoloring methyl orange (MeOr) under sunlight.

\section{Materials and Methods}

2.1. Materials. Benzophenone (BP), 2-hydroxyethyl methacrylate (HEMA), methyl orange (MeOr), $\mathrm{Zn}\left(\mathrm{NO}_{3}\right)_{2}$, and oxalic acid were used as purchased from Lingfeng Shanghai Reagent Co. Ltd. Polypropylene macroporous membranes (PPMMs) with a porosity of $45-50 \%$ and an average pore diameter of $0.10 \mu \mathrm{m}$ were prepared [21], and benzyl dithiobenzoate (BDTB) was synthesized according to the literature [22].

2.2. Synthesis of $\mathrm{ZnONPs}$. $\mathrm{ZnONPs}$ were synthesized by direct precipitation with oxalic acid as the precipitator [23] with minor modification. $12.7 \mathrm{~g} \mathrm{Zn}\left(\mathrm{NO}_{3}\right)_{2}$ and $10.0 \mathrm{~g}$ oxalic acid were dissolved in $50 \mathrm{~mL}$ distilled water separately. Then the two solutions were mixed slowly under stirring during which the white precipitate produced immediately. After this, the precipitate was washed with distilled water and ethanol for three or four times, dried at $90-100^{\circ} \mathrm{C}$, and finally calcined at $450^{\circ} \mathrm{C}$ for $3 \mathrm{~h}$ to achieve $\mathrm{ZnO}$ NPs.

2.3. Surface Modification of Membrane. The used method and experimental setup for the membrane surface modification were described in [19].

2.4. Photocatalytic Membrane Reactor. The permeation properties of the unmodified and modified PPMMs were examined in a stirred dead-ended ultrafiltration test cell connected to a $2 \mathrm{~L}$ feed tank [24]. The volumetric flux was determined through the timed collection of permeate, and the relative flux was described by the following equation:

$$
\text { Relative flux }=\frac{J_{0, m}}{J_{0, u}} \times 100 \% \text {, }
$$

where $J_{0, u}$ and $J_{0, m}$ are the pure water fluxes through the unmodified and modified membranes. $J_{0, u}$ varied from 250 to $450 \mathrm{Lm}^{-2} \mathrm{~h}^{-1}$, and as a result, the relative flux was adopted to eliminate the differences between the unmodified membranes. 
The PHEMA grafted PPMMs were submerged in the $\mathrm{ZnO}$ NPs ethanol dispersion for $24 \mathrm{~h}$; ZnO NPs were physically adsorbed on the membrane surface [24]. The immobilization degree (ID) of the ZnO NPs on the membrane is calculated by the following equation:

$$
\mathrm{ID}=\frac{\left(W_{2}-W_{1}\right)}{W_{1}} \times 100 \%,
$$

where ID refers to the immobilization degree of $\mathrm{ZnO}$ NPs on the membrane surface, $\%(\mathrm{wt})$, and $W_{1}$ and $W_{2}$ the respective weights of PHEMA grafted membranes before and after the immobilization of the $\mathrm{ZnO} N P$ s. All the results are the average values of three parallel experiments.

The photocatalytic decoloring MeOr with ZnO NPs suspended is investigated. $0.05 \mathrm{~g} \mathrm{ZnO} \mathrm{NPs} \mathrm{were} \mathrm{added} \mathrm{to} 200 \mathrm{~mL}$ $10 \mathrm{mg} / \mathrm{L} \mathrm{MeOr}$ aqueous solution, stirred for $30 \mathrm{~min}$ in the dark to achieve the adsorption-desorption equilibrium, and then exposed to the sunlight with stirring (Wuhu, Anhui Province, China, July 2010) (the light intensity was determined by a UV power meter, LS123, Shenzhen Linshang Technology Co. Ltd.); the photocatalytic reaction was conducted within the light intensity of $90 \pm 15 \mathrm{~mW} / \mathrm{cm}^{2}$; then pure water was added to the reacting solution to compensate the evaporation during its exposition to sunlight. The solution was sampled at intervals of $5 \mathrm{~min}$, and the samples were centrifuged for $10 \mathrm{~min}$.

The PMR with ZnO NPs immobilized on the membrane surface is similar to [24]. The membrane (GD $=36.0 \%$, ID $=6.2 \%$ ) was sandwiched in the cross flow PMMA reactor (each of the PMMA plate is $20 \mathrm{~mm}$ in thickness), the total weight of the immobilized ZnO NPs is about $0.05 \mathrm{~g} ; 200 \mathrm{~mL}$ $10 \mathrm{mg} / \mathrm{L} \mathrm{MeOr}$ aqueous solution was pumped into the reactor circularly by a peristaltic pump with the reactor exposed to the sunlight, and the cross flow velocity was kept at $0.15 \mathrm{~m} / \mathrm{s}$ [25]. At the end of each run, the membrane was taken out, flushed with pure water for three times, then dried and stored in air for a period of time (as shown in Figure 6). After that the next run started.

The supernatant of the effluent was measured by the UV-Vis spectrophotometer (UV-2450, Shimadzu, Japan) at $464 \mathrm{~nm}$ (the maximal adsorption wavelength of MeOr). The photocatalytic decoloration percentage (PDP) is calculated by the following equation:

$$
\mathrm{PDP}=\frac{A_{0}-A}{A_{0}} \times 100 \%,
$$

where $A$ and $A_{0}$ correspond to the UV absorbance of the feed and the filtrate, respectively.

2.5. Characterization. The crystalline structures of $\mathrm{ZnO}$ NPs were examined by X-ray powder diffraction on an X-ray diffractometer (XRD) (XRD-6000, Japan) with $\mathrm{CuK} \alpha$ radiation $(\lambda=0.154056 \mathrm{~nm})$ at a scanning rate of $0.05 \%$ s. Surface morphology of the membranes was observed by field emission scanning electron microscopy (FESEM) (Hitachi 4800, Japan), and the surface hydrophilicity was determined by water contact angle measurements (OCA20, Germany) [26].

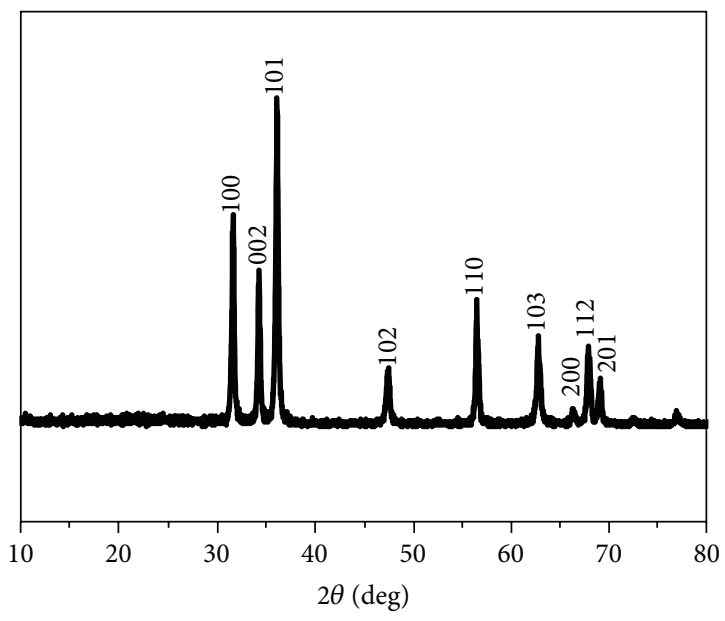

FIGURE 2: XRD pattern of ZnO NPs.

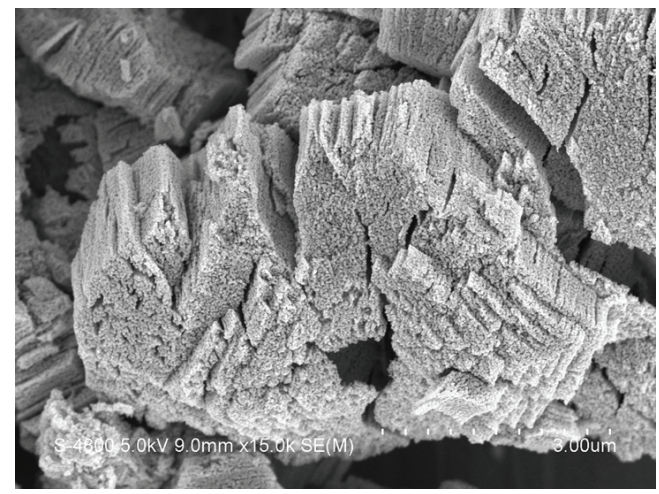

FIGURE 3: FESEM image of the ZnO NPs $(\times 15000)$.

\section{Results and Discussion}

3.1. Characterization and Permeation of the Membranes. XRD is used to investigate the changes of crystalline nature of the prepared $\mathrm{ZnO}$ NPs (Figure 2). Most of the diffraction peaks could be attributed to the hexagonal wurtzite structure of $\mathrm{ZnO}$ (JCPDS card 36-1451) [27]. It can be observed that no diffraction peaks from other phases of $\mathrm{ZnO}$ or impurities, suggesting that the obtained $\mathrm{ZnO}$ is of high purity. The average crystallite size for $\mathrm{ZnO}$ NPs calcined at $450^{\circ} \mathrm{C}$ was calculated at $26.6 \pm 4.8 \mathrm{~nm}$ according to the Scherrer formula [28].

Figure 3 presents the FESEM images of the ZnONPs, which reveal that the surfaces are rough and porous, indicating that the $\mathrm{ZnO} N$ Ps aggregate due to large specific surface area and high surface energy.

The surface morphologies of the unmodified, the PHEMA grafted, and the ZnO NPs immobilized membranes were observed by FESEM (Figure 4). Compared with the unmodified PPMM with high porosity (Figure 4(a)), the surfaces of the PHEMA grafted PPMMs are gradually covered with polymers; as the grafting degree increases (Figures 4(b) and 4(c)), the membrane pores are plugged and the surface porosity reduces with GD increasing. It can be clearly observed that $\mathrm{ZnO}$ NPs have been introduced to the 
TABLE 1: Relative water flux (\%) through PHEMA grafted and ZnO NPs immobilized PPMMs with different GD. The average water flux for the membranes is $400 \pm 25 \mathrm{Lm}^{-2} \mathrm{~h}^{-1}$.

\begin{tabular}{lccc}
\hline GD, \% (wt) & Water contact angle on PHEMA grafted PPMMs, & \multicolumn{2}{c}{ Relative water flux, \% } \\
& & ZnO NPs immobilized PPMM \\
\hline 0.00 & 90 & 100 & 113 \\
1.00 & 72 & 120 & 124 \\
4.48 & 54 & 118 & 143 \\
6.89 & 43 & 115 & - \\
8.21 & 35 & 116 & 136 \\
\hline
\end{tabular}

-: The membrane was broken after ZnO NPs immobilization. The error margin is within $\pm 10 \%$.

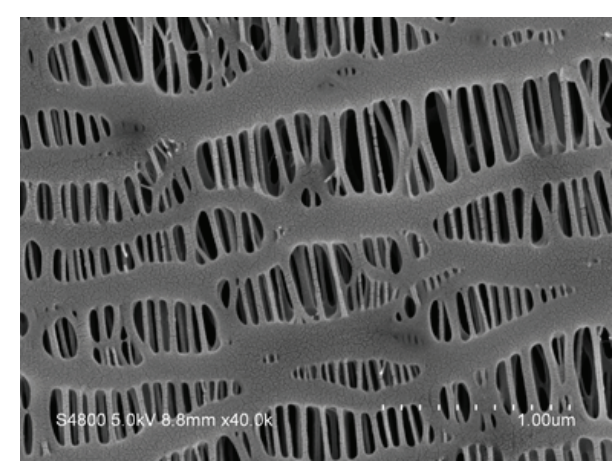

(a)

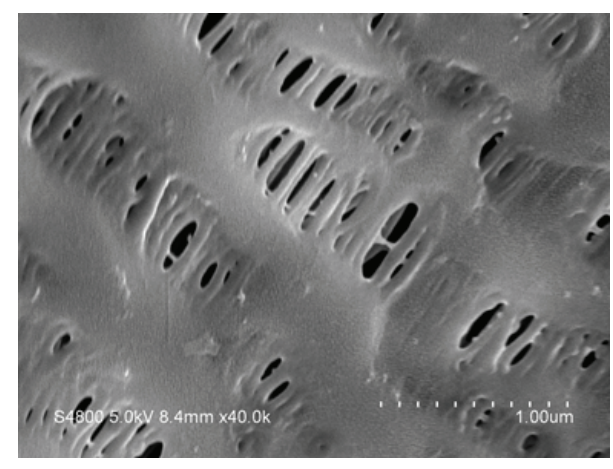

(c)

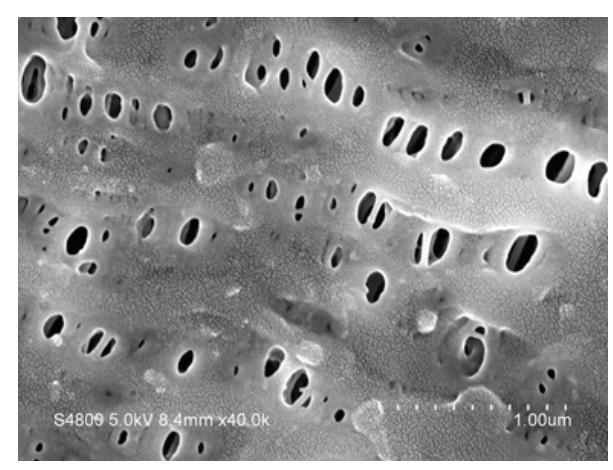

(b)

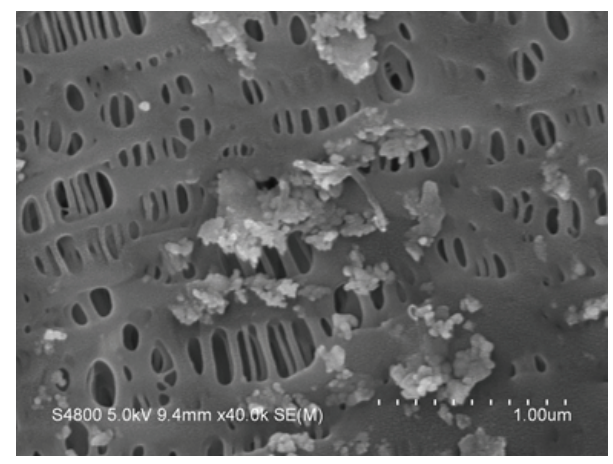

(d)

FIGURE 4: FESEM images ( $\times 40000$ ) of (a) the unmodified, and (b)-(c) PHEMA grafted PPMMs with GD = 2.40 and $4.05 \%$ (wt), respectively; (d) ZnO NPs immobilized PPMMs with GD $=36.0 \%$ (wt), ID $=6.2 \%$ (wt).

membrane surface from Figure 4(d). The ZnO NPs appear in large clusters, instead of being evenly distributed. The immobilization degree of $\mathrm{ZnO}$ NPs immobilized PPMMs with different GD of PHEMA was shown in Supplementary Material (See Supplementary Material available online at http://dx.doi.org/10.1155/2013/451398). The result verified that the grafting of polyHEMA can enhance the adsorption of $\mathrm{ZnO}$ NPs on the hydrophobic membrane surface.

Variation of the relative water fluxes through PHEMA grafted and ZnO NPs immobilized PPMMs was investigated (Table 1). The relative water flux goes up after PHEMA grafted on the PPMMs due to the membrane surface hydrophilicity increasing (Table 1) [29]. For the PHEMA grafted membrane with a GD of $4.48 \%$ (wt), the relative water flux reaches the maximum value of $118 \%$; after that, the relative water flux decreases slowly.
Membrane permeability in the filtration of aqueous solution is mainly determined by two factors: one is the membrane structure, such as membrane thickness, pore size, and porosity; the other is the membrane surface hydrophilicity. When DG is low, the hydration of PHEMA chains results in a decreased resistance to water permeation and thus improves the water fluxes. For the membranes with a high DG, the graft layer is thick and the expanded PHEMA conformation in aqueous circumstance tends to cover the membrane pores, and consequently the water fluxes decrease [30, 31].

It can also be found in Table 1 that after the immobilization of $\mathrm{ZnO}$ NPs on the membrane surface, the membrane permeability continues to increase and the relative water flux rises to $143 \%$. The coating of $\mathrm{ZnO}$ NPs on the membrane causes the decrease of water contact angle, because hydrophilic $\mathrm{ZnO} N \mathrm{~N}$ anchored to the membrane [32, 33], 


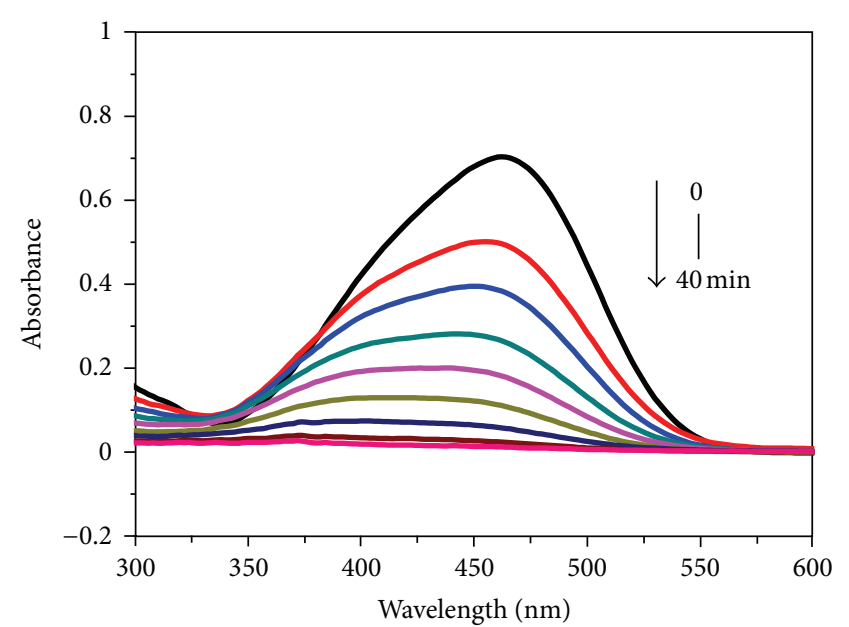

FIGURE 5: UV-Vis spectrum of MeOr under sunlight, using the photocatalytic membrane reactor with the $\mathrm{ZnO}$ NPs in suspension.

leading to the increase of the membrane surface hydrophilicity and hence increases the membrane permeability.

For the filtration of the dye solution, it was observed that the relative water fluxes were similar to those of the pure water fluxes, that is, the dyes could not be rejected by the membranes, and as a result, the filtrate was returned to the feed tank for the improvement of dyes decoloration. Membranes just acted as barriers for the $\mathrm{ZnO}$ NPs.

3.2. Photocatalytic Decoloring MeOr under Sunlight. In order to examine the performance of $\mathrm{ZnONPs}$ in decoloring dyes in wastewater, photocatalytically decoloring $\mathrm{MeOr}$ was carried out in a photocatalytic membrane reactor under sunlight. The catalysts were suspended in the solution or immobilized on the membrane.

Figure 5 shows that MeOr is nearly completely decolored after 40 min sunlight (ZnO NPs were suspended in the solution). The result shows that $\mathrm{ZnO}$ NPs prepared in the present work are highly effective in decoloring dyes in water and wastewater. The mechanism of $\mathrm{ZnO}$ NPs catalyzed decoloring reaction is as follows. ZnO NPs are excited by absorbing the photon with more energy than the band gap, resulting to the photoinduced electron-hole pairs. The peroxy radical anions are reacted by the electron and oxygen, and the hydroxyl radical is reacted by the hole and water. Hydroxyl radical can further decompose and remove organic molecules, namely, organic photodegradation [34]. Figure 5 also shows that the peak position is blue-shifted along with reaction time, which may be attributed to the deamination products of $\mathrm{MeOr}$ $[35,36]$.

The photostability of $\mathrm{ZnO}$ NPs photocatalysts is an essential factor to promote heterogeneous photocatalysis technology for practical applications. In this research, hybrid membrane is derived from coating ZnO NPs on the PHEMA grafted membrane. The photocatalytic reaction was conducted within the light intensity of $90 \pm 15 \mathrm{~mW} / \mathrm{cm}^{2}$ for each run to eliminate the differences in light intensity. After each run, the membrane was taken out of the reaction chamber,

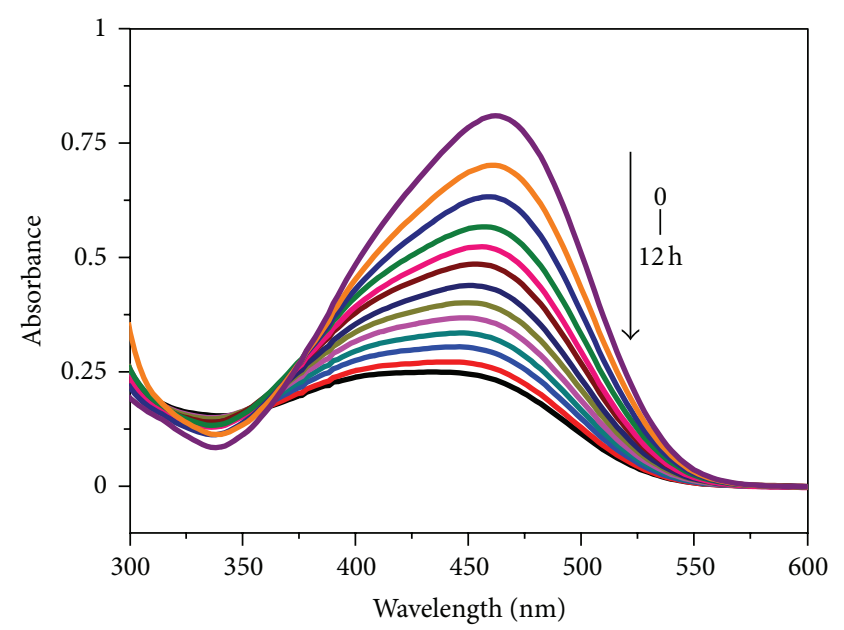

(a)

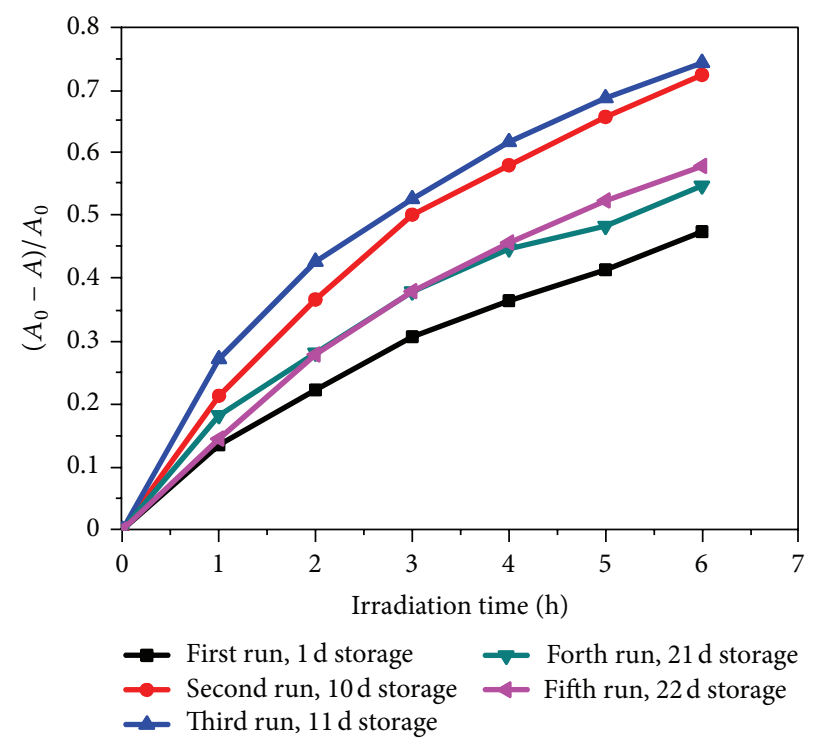

(b)

FIGURE 6: Decoloring MeOr in the PMR with the ZnO NPs immobilized on the membrane surface. (a) UV-Vis spectrum of MeOr at $1 \mathrm{~h}$ intervals (1-12 h) for the first cycle; (b) photocatalytic experiments for 5 cycles.

flushed with pure water and dried in air. UV-Vis spectrum of $\mathrm{MeOr}$ at $1 \mathrm{~h}$ intervals $(1-12 \mathrm{~h}$ ) for the first cycle is depicted in Figure 6(a), which shows that MeOr was decolored slowly. The photostability of ZnO NPs immobilized membrane for the decoloring MeOr is shown in Figure 6(b), which indicates that the $\mathrm{ZnO}$ NPs immobilized on polypropylene membrane can photocatalytically decolor MeOr by $47.4 \%, 72.4 \%, 74.3 \%$, $54.7 \%$, and $57.8 \%$ after $6 \mathrm{~h}$ sunlight irradiation from the first to the fifth run, respectively. The PDP of the first run is the lowest, but those of the second and the third run reach the highest.

The reason may be that the active point of $\mathrm{ZnONPs}$ was covered because of the agglomeration. After the first run, the agglomeration configuration of $\mathrm{ZnONPs}$ became 
a little dispersive and the MeOr can enter into the interstice between $\mathrm{ZnONPs.} \mathrm{In} \mathrm{addition,} \mathrm{the} \mathrm{transparence} \mathrm{of}$ membrane increased after the first run, and the loosely adsorbed $\mathrm{ZnO}$ NPs were washed off (however, this could not be determined due to the trivial gravity changes), causing an increase in sunlight penetrating, hence the PDP ascends.

PDPs of the fourth and fifth run are between those of the first and the second run, showing that PDPs of the two runs slightly decrease, compared with those of the second and the third run. This phenomenon may be resulted from the deactivation of the photocatalysts, which is due to that many MeOr molecules were adsorbed on the membrane surface after being used for three times [37]. The PDP maintains at a very high level of about 55\% in the fifth run, which means that after being used for five cycles, the PDP is still $8 \%$ higher than that of the first run. This result confirms that the catalysts were not photocorroded during the photocatalytic decoloration of the pollutant molecules under sunlight. After being used for five times, a lot of $\mathrm{ZnO}$ NPs were also visibly adsorbed on the membrane surface, suggesting that they are not easy to wash off.

These results demonstrate that PMR with $\mathrm{ZnONPs}$ immobilized on the polypropylene macroporous membrane surface can be reused for several times without weakening the photocatalytic activity, exhibiting potential application for continual and long-time reactions [38]. Generally, the immobilized $\mathrm{ZnO}$ NPs are stable for repeated uses [34]. Therefore, the $\mathrm{ZnONPs}$ can be regarded as ideal photocatalysts for environmental friendly purification on industrial scale under sunlight.

Figure 6 shows that the PDPs are much lower than those of the PMR with ZnO NPs in suspension (Figure 5), which may be attributed to the following reasons: (1) the active area of $\mathrm{ZnO}$ NPs decreased; (2) 3/4 of the sunlight was absorbed by the PMMA reactor, which was characterized by a UV power meter; consequently, a more effective reactor with little absorption of the sunlight should be adopted.

It is well known that the polymeric membranes are not promising materials for application in the PMRs due to their susceptibility to damage by irradiation and hydroxyl radicals [24]. However, in the present work, sunlight was employed to decompose the dye; the damage effect was infinitesimal, and as a result, the mechanical properties were not presented.

\section{Conclusions}

Decoloring methyl orange in a photocatalytic membrane reactor was conducted via coupling zinc oxide nanoparticles and polypropylene macroporous membrane under sunlight. Poly(2-hydroxyethyl methacrylate) was grafted on the membrane surface to enhance the physical adsorption of zinc oxide nanoparticles on the membrane and to improve the membrane permeability.

The poly(2-hydroxyethyl methacrylate) modified membrane with a grafting degree of $4.48 \%$ (wt) has the maximum value, the relative flux is $118 \%$; after the immobilization of zinc oxide nanoparticles on this membrane surface, it reaches $143 \%$, improved by $25 \%$.
The photocatalytic decoloration percent of methyl orange is $98.2 \%$ under $40 \mathrm{~min}$ sunlight irradiation in the photocatalytic membrane reactor with the ZnO NPs in suspension; and in the photocatalytic membrane reactor with the $\mathrm{ZnO}$ NPs immobilized on the membrane, the photocatalytic decoloration percent of methyl orange decreases greatly; it reaches $74.3 \%$ under $6 \mathrm{~h}$ sunlight irradiation. Overall, after repeated uses for five times, it is still $8 \%$ higher than that of the first run. These results demonstrate that the immobilized $\mathrm{ZnO}$ NPs are stable for repeated uses.

\section{Nomenclature}

$\begin{array}{ll}\text { BDTB: } & \text { Benzyl dithiobenzoate } \\ \text { BP: } & \text { Benzophenone } \\ \text { GD: } & \text { Grafting degree } \\ \text { ID: } & \text { Immobilization degree } \\ \text { FESEM: } & \text { Field emission scanning electron } \\ & \text { microscopy } \\ \text { HEMA: } & \text { 2-hydroxyethyl methacrylate } \\ \text { PHEMA: } & \text { Poly-2-hydroxyethyl methacrylate } \\ \text { MeOr: } & \text { Methyl orange } \\ \text { PDPs: } & \text { Photocatalytic decoloration percents } \\ \text { PMR: } & \text { Photocatalytic membrane reactor } \\ \text { PPMM: } & \text { Polypropylene macroporous membrane } \\ \text { RAFT: } & \text { Reversible addition-fragmentation } \\ & \text { chain transfer } \\ \text { ZnO NPs: } & \text { Zinc oxide nanoparticles. }\end{array}$

\section{Authors' Contribution}

B. $\mathrm{Hu}$ contributed to the preparation and characterization of $\mathrm{ZnO}$ NPs and membrane surface modification. X.-M. Wu contributed to the synthesis of the chain transfer agent. J. Zhou, corresponding author, contributed to the conducting photocatalytic membrane reactor and photocatalytic decoloring of dye.

\section{Acknowledgments}

We gratefully acknowledge the financial support from the National Natural Science Foundation of China (No. 20871003) and the instruction of professor Hai-Yin Yu.

\section{References}

[1] A. Akbari, J. C. Remigy, and P. Aptel, "Treatment of textile dye effluent using a polyamide-based nanofiltration membrane," Chemical Engineering and Processing, vol. 41, no. 7, pp. 601-609, 2002.

[2] V. K. Gupta, R. Jain, A. Mittal, M. Mathur, and S. Sikarwar, "Photochemical degradation of the hazardous dye Safranin-T using $\mathrm{TiO}_{2}$ catalyst," Journal of Colloid and Interface Science, vol. 309, no. 2, pp. 464-469, 2007.

[3] V. K. Gupta, R. Jain, A. Nayak, S. Agarwal, and M. Shrivastava, "Removal of the hazardous dye-Tartrazine by photodegradation on titanium dioxide surface," Materials Science and Engineering C, vol. 31, no. 5, pp. 1062-1067, 2011. 
[4] V. K. Gupta, R. Jain, A. Mittal et al., "Photo-catalytic degradation of toxic dye amaranth on $\mathrm{TiO}(2) / \mathrm{UV}$ in aqueous suspensions," Materials Science and Engineering C, vol. 32, no. 1, pp. $12-17,2012$.

[5] V. K. Gupta, R. Jain, S. Agarwal, A. Nayak, and M. Shrivastava, "Photodegradation of haz- ardous dye quinoline yellow catalyzed by $\mathrm{TiO}_{2}$," Journal of Colloid and Interface Sciences, vol. 366, no. 1, pp. 135-140, 2012.

[6] V. K. Gupta, R. Jain, S. Agarwal, and M. Shrivastava, "Kinetics of photo-catalytic degradation of hazardous dye Tropaeoline 000 using $\mathrm{UV} / \mathrm{TiO}_{2}$ in a UV reactor," Colloids and Surfaces $A$, vol. 378, no. 1-3, pp. 22-26, 2011.

[7] A. Lei, B. Qu, W. Zhou, Y. Wang, Q. Zhang, and B. Zou, "Facile synthesis and enhanced photocatalytic activity of hierarchical porous $\mathrm{ZnO}$ microspheres," Materals Letters, vol. 66, no. 1, pp. 72-75, 2012.

[8] R. Mohan, K. Krishnamoorthy, and S. J. Kim, "Enhanced photocatalytic activity of $\mathrm{Cu}$-doped $\mathrm{ZnO}$ nanorods," Solid State Communications, vol. 152, no. 5, pp. 375-380, 2012.

[9] R. Selvin, H. L. Hsu, N. S. Arul, and S. Mathew, "Comparison of photo-catalytic efficiency of various metal oxide photo-catalysts for the degradation of methyl orange," Science of Advanced Materials, vol. 2, no. 1, pp. 58-63, 2010.

[10] Y. Abdollahi, A. H. Abdullah, Z. Zainal, and N. A. Yusof, "Photodegradation of $\mathrm{m}$-cresol by zinc oxide under visible-light irradiation," International Journal of Chemistry, vol. 3, no. 3, pp. 31-43, 2011.

[11] Y. Abdollahi, A. H. Abdullah, Z. Zainal, and N. A. Yusof, "Photocatalytic Degradation of p-Cresol by Zinc Oxide under UV Irradiation," International Journal of Molecular Sciences, vol. 13, no. 1, pp. 302-315, 2012.

[12] Y. Gou, D. Chen, and Z. Su, "Photocatalyst of nanometer $\mathrm{TiO}_{2} /$ conjugated polymer complex employed for depigmentation of methyl orange," Applied Catalysis A, vol. 261, no. 1, pp. 15-18, 2004.

[13] H. R. Pouretedal and M. H. Keshavarz, "Synthesis and characterization of $\mathrm{Zn}_{1-X} \mathrm{Cu}_{X} \mathrm{~S}$ and $\mathrm{Zn1-XNiXS}$ nanoparticles and their applications as photocatalyst in Congo red degradation," Journal of Alloys and Compounds, vol. 501, no. 1, pp. 130-135, 2010.

[14] M. Zhang, L. Zhang, L. H. Cheng et al., "Extracorporeal endotoxin removal by novel l-serine grafted PVDF membrane modules," Journal of Membrane Science, vol. 405, no. 1, pp. 104112, 2012.

[15] R. A. Damodar, S. J. You, and G. W. Chiou, "Investigation on the conditions mitigating membrane fouling caused by $\mathrm{TiO}_{2}$ deposition in a membrane photocatalytic reactor (MPR) used for dye wastewater treatment," Journal of Hazardous Materals, vol. 203, no. 15, pp. 348-356, 2013.

[16] G. Zhang, J. Zhang, L. Wang, Q. Meng, and J. Wang, "Fouling mechanism of low-pressure hollow fiber membranes used in separating nanosized photocatalysts," Journal of Membrane Science, vol. 389, no. 1, pp. 532-543, 2012.

[17] J. Marto, P. S. Marcos, T. Trindade, and J. A. Labrincha, "Photocatalytic decolouration of Orange II by $\mathrm{ZnO}$ active layers screen-printed on ceramic tiles," Journal of Hazardous Materials, vol. 163, no. 1, pp. 36-42, 2009.

[18] S. Rehman, R. Ullah, A. M. Butt, and N. D. Gohar, "Strategies of making $\mathrm{TiO}_{2}$ and $\mathrm{ZnO}$ visible light active," Journal of Hazardous Materials, vol. 170, no. 2-3, pp. 560-569, 2009.
[19] H. Y. Yu, J. Zhou, J. S. Gu, and S. Yang, "Manipulating membrane permeability and protein rejection of UV-modified polypropylene macroporous membrane," Journal of Membrane Science, vol. 364, no. 1-2, pp. 203-210, 2010.

[20] B. Hu, L. Wang, X. M. Wu, S. Yang, J. S. Gu, and H. Y. Yu, "Low protein fouling polypropylene membrane prepared by photoinduced reversible addition-fragmentation chain transfer polymerization," Journal of Applied Polymer Science, vol. 123, no. 6, pp. 3668-3674, 2011.

[21] H. Y. Yu, Z. K. Xu, Q. Yang, M. X. Hu, and S. Y. Wang, "Improvement of the antifouling characteristics for polypropylene microporous membranes by the sequential photoinduced graft polymerization of acrylic acid," Journal of Membrane Science, vol. 281, no. 1-2, pp. 658-665, 2006.

[22] J. Chiefari, Y. K. Chong, F. Ercole et al., "Living free-radical polymerization by reversible addition-fragmentation chain transfer: the RAFT process," Macromolecules, vol. 31, no. 16, pp. 5559$5562,1998$.

[23] D. Zhang, H. Xu, M. Xue, W. Xu, and V. Tarasov, "Preparation and photocatalytic kinetics of nano- $\mathrm{ZnO}$ powders by precipitation stripping process," Frontiers of Chemical Engineering in China, vol. 2, no. 3, pp. 319-324, 2008.

[24] S. Yang, J. S. Gu, H. Y. Yu et al., "Polypropylene membrane surface modification by RAFT grafting polymerization and $\mathrm{TiO}_{2}$ photocatalysts immobilization for phenol decomposition in a photocatalytic membrane reactor," Separation and Purification Technology, vol. 83, no. 15, pp. 157-165, 2011.

[25] T. Toshinori, K. Takehiro, Y. Tomohisa, and A. Masashi, "A photocatalytic membrane reactor for VOC decomposition using Pt-modified titanium oxide porous membranes," Journal of Membrane Science, vol. 280, no. 1-2, pp. 156-162, 2006.

[26] J. S. Gu, H. Y. Yu, L. Huang et al., "Chain-length dependence of the antifouling characteristics of the glycopolymer-modified polypropylene membrane in an SMBR," Journal of Membrane Science, vol. 326, no. 1, pp. 145-152, 2009.

[27] R. Brayner, S. A. Dahoumane, C. Yéprémian et al., “ $\mathrm{ZnO}$ nanoparticles: synthesis, characterization, and ecotoxicological studies," Langmuir, vol. 26, no. 9, pp. 6522-6528, 2010.

[28] R. Hong, T. Pan, J. Qian, and H. Li, "Synthesis and surface modification of $\mathrm{ZnO}$ nanoparticles," Chemical Engineering Journal, vol. 119, no. 2-3, pp. 71-81, 2006.

[29] M. X. Hu, Q. Yang, and Z.-K. Xu, "Enhancing the hydrophilicity of polypropylene microporous membranes by the grafting of 2hydroxyethyl methacrylate via a synergistic effect of photoinitiators," Journal of Membrane Science, vol. 285, no. 1-2, pp. 196205, 2006.

[30] D. S. Wavhal and E. R. Fisher, "Membrane surface modification by plasma-induced polymerization of acrylamide for improved surface properties and reduced protein fouling," Langmuir, vol. 19, no. 1, pp. 79-85, 2003.

[31] Q. Yang, Z. K. Xu, Z. W. Dai, J. L. Wang, and M. Ulbricht, "Surface modification of polypropylene microporous membranes with a novel glycopolymer," Chemistry of Materials, vol. 17, no. 11, pp. 3050-3058, 2005.

[32] C. Y. Kuan, M. H. Hon, J. M. Chou, and I. C. Leu, "Wetting characteristics on micro/nanostructured zinc oxide coatings," Journal of the Electrochemical Society, vol. 156, no. 2, pp. J32J36, 2009.

[33] O. Akhavan, M. Mehrabian, K. Mirabbaszadeh, and R. Azimi$\mathrm{rad}$, "Hydrothermal synthesis of $\mathrm{ZnO}$ nanorod arrays for photocatalytic inactivation of bacteria," Journal of Physics D, vol. 42, no. 22, Article ID 225305, 2009. 
[34] G. Wang, D. Chen, H. Zhang, J. Z. Zhang, and J. Li, “Tunable photocurrent spectrum in well-oriented zinc oxide nanorod arrays with enhanced photocatalytic activity," Journal of Physical Chemistry C, vol. 112, no. 24, pp. 8850-8855, 2008.

[35] R. Y. Hong, J. H. Li, L. L. Chen et al., "Synthesis, surface modification and photocatalytic property of $\mathrm{ZnO}$ nanoparticles," Powder Technology, vol. 189, no. 3, pp. 426-432, 2009.

[36] F. B. Li, G. B. Gu, G. F. Huang, Y. L. Gu, and H. F. Wan, " $\mathrm{TiO}_{2}$ assisted photo-catalysis degradation process of dye chemicals," Journal of Environmental Sciences, vol. 13, no. 1, pp. 64-68, 2001.

[37] J. Liqiang, X. Baifu, Y. Fulong et al., "Deactivation and regeneration of $\mathrm{ZnO}$ and $\mathrm{TiO}_{2}$ nanoparticles in the gas phase photocatalytic oxidation of $n-\mathrm{C}_{7} \mathrm{H}_{16}$ or $\mathrm{SO}_{2}$," Applied Catalysis A, vol. 275, no. 1-2, pp. 49-54, 2004.

[38] J. Xu, Y. Ao, D. Fu, and C. Yuan, "Low-temperature preparation of F-doped $\mathrm{TiO}_{2}$ film and its photocatalytic activity under solar light," Applied Surface Science, vol. 254, no. 10, pp. 3033-3038, 2008. 

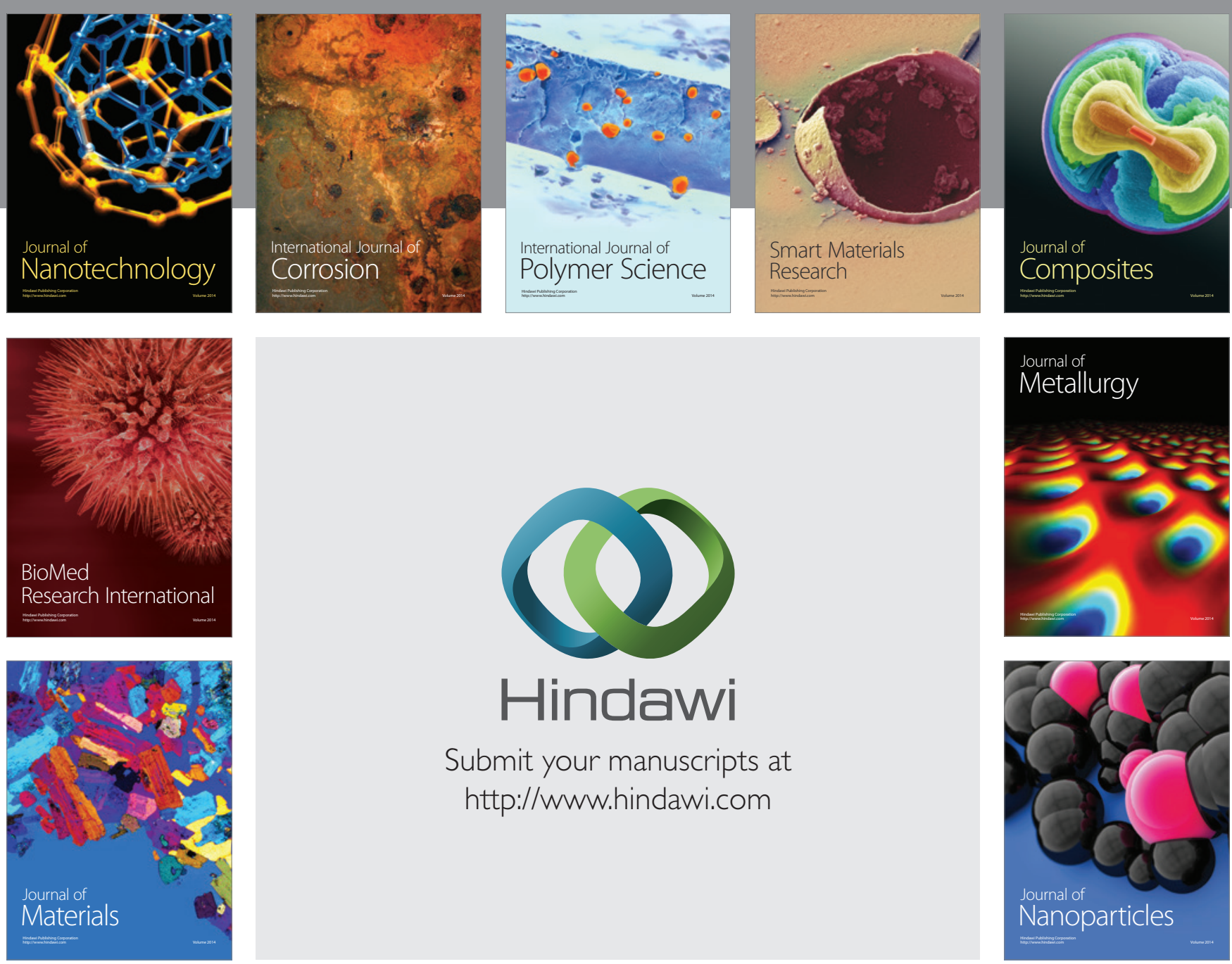

Submit your manuscripts at http://www.hindawi.com
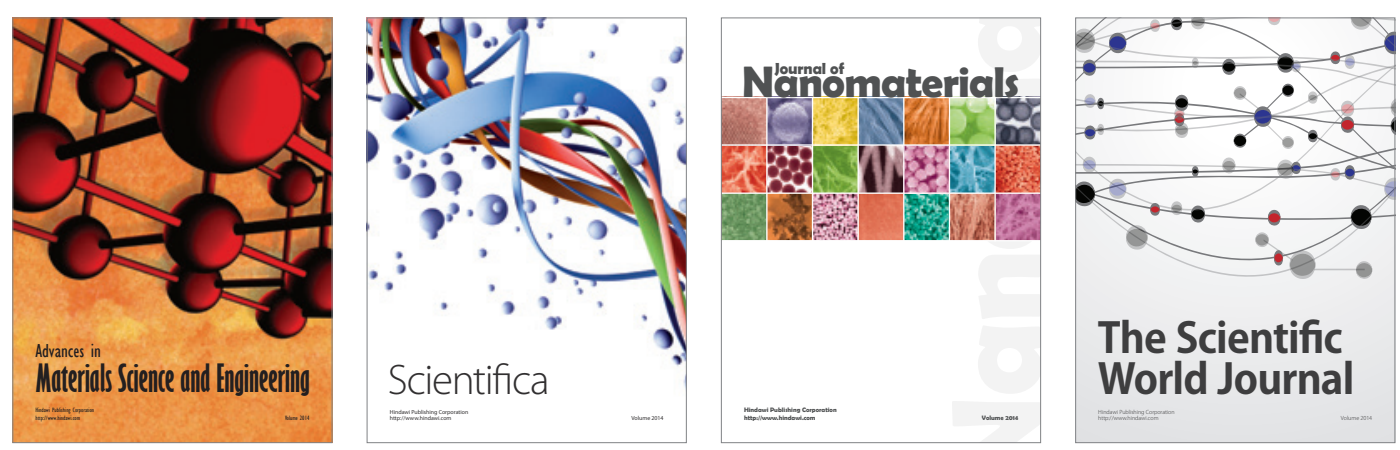

\section{The Scientific World Journal}
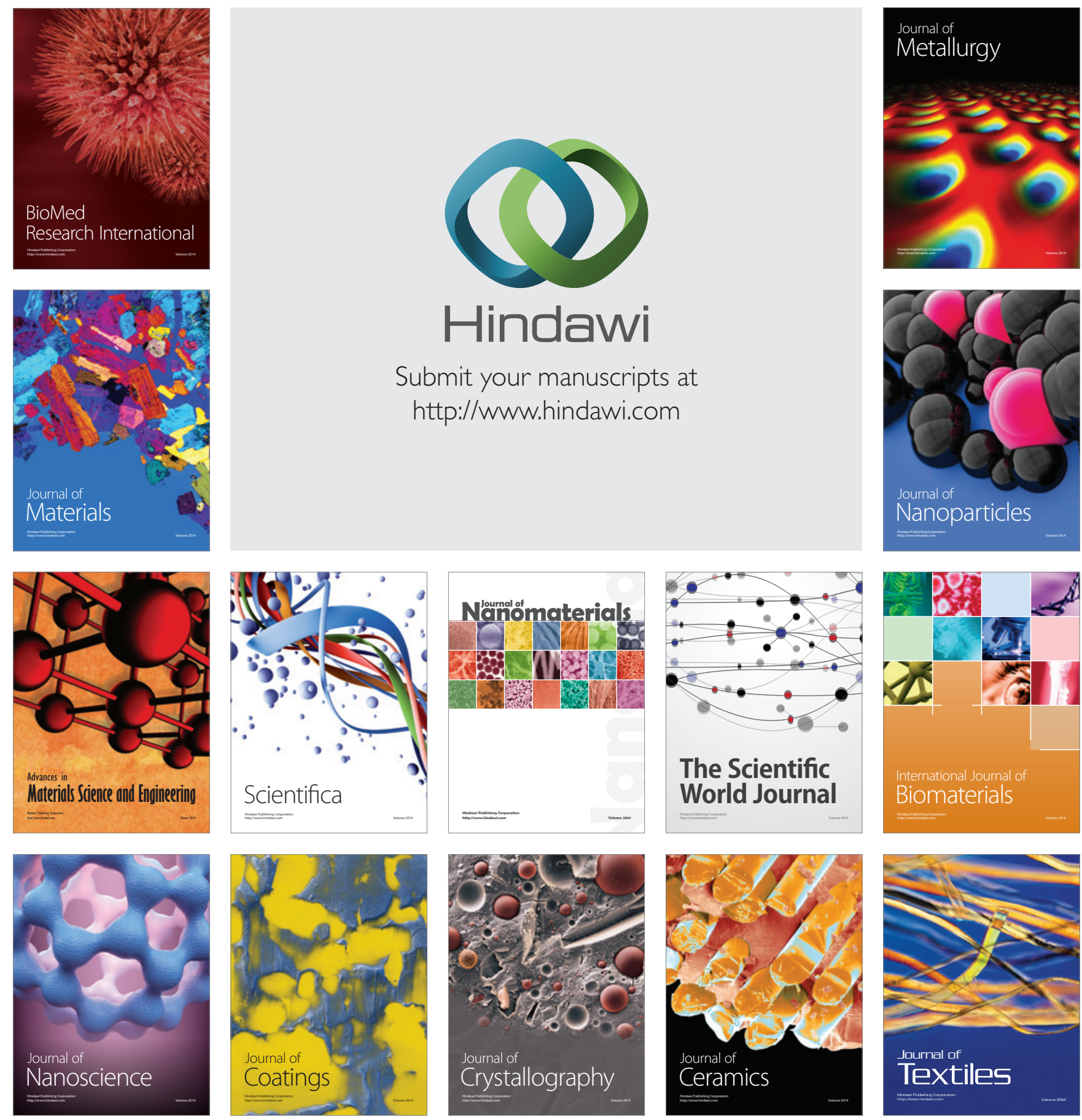